\title{
Maize Centromere Mapping: A Comparison of Physical and Genetic Strategies
}

\author{
Ron j. Okagaki, Morrison S. Jacobs, Adrian O. Stec, Ralf G. Kynast, Elizabeth Buescher, \\ Howard W. Rines, M. Isabel Vales, Oscar Riera-lizarazu, Martha Schneerman, Greg Doyle, \\ Katherine L. Friedman, Rick W. Staub, David F. Weber, Terry L. Kamps, ina F. E. Amarillo, \\ Christine D. Chase, Hank W. Bass, and Ronald L. Phillips
}

From the Department of Agronomy and Plant Genetics, University of Minnesota, St Paul, MN 55 I 08-6026 (Okagaki, Jacobs, Stec, Kynast, Buescher, Rines, and Phillips); Plant Science Research Unit, the United States Department of AgricultureAgricultural Research Service, St. Paul, MN 55 I 08 (Rines); the Department of Crop and Soil Sciences, Oregon State University, Corvallis, OR 9733 I-3002 (Vales and Riera-Lizarazu); the Department of Biological Sciences, Illinois State University, Normal, IL 61790-4I 20 (Schneerman and Weber); the Department of Agronomy, University of Missouri, Columbia, MO 6521 I-7020 (Doyle); the Department of Biology, Carleton College, Northfield, MN 55057 (Friedman and Staub); the Horticultural Sciences Department, University of Florida, Gainesville, FL 326 I I-0690 (Kamps and Chase); and the Department of Biological Science, Florida State University, Tallahassee, FL 32306-4370 (Amarillo and Bass). Morrison S. Jacobs is now at the Monsanto Co., 800 North Lindbergh Boulevard, St Louis, MO 63167; Ralf G. Kynast is now at the Jodrell Laboratory, Royal Botanic Gardens, Kew, Surrey, TW9 3AB, UK; Elizabeth Buescher is now at the Agronomy Department, Purdue University, West Lafayette, IN 47907-I I50; Martha Schneerman is now at the Division of Research Safety, University of Illinois, Urbana, IL 6 I 80 I ; Katherine L. Friedman is now at the Department of Biological Sciences, Vanderbilt University, Nashville, TN 37235; and Rick W. Staub is now at the Orchid Cellmark Inc., I3988 Diplomat Drive, Suite 100, Dallas, TX 75234.

Address correspondence to R. J. Okagaki at the address above, or e-mail: okaga002@umn.edu.

\begin{abstract}
Centromere positions on 7 maize chromosomes were compared on the basis of data from 4 to 6 mapping techniques per chromosome. Centromere positions were first located relative to molecular markers by means of radiation hybrid lines and centric fission lines recovered from oat-maize chromosome addition lines. These centromere positions were then compared with new data from centric fission lines recovered from maize plants, half-tetrad mapping, and fluorescence in situ hybridizations and to data from earlier studies. Surprisingly, the choice of mapping technique was not the critical determining factor. Instead, on 4 chromosomes, results from all techniques were consistent with a single centromere position. On chromosomes 1, 3, and 6, centromere positions were not consistent even in studies using the same technique. The conflicting centromere map positions on chromosomes 1, 3, and 6 could be explained by pericentric inversions or alternative centromere positions on these chromosomes.
\end{abstract}

Centromeres are a fundamental structural feature of eukaryotic chromosomes that are the obvious reference points for linking cytological, molecular, and genetic maps. Techniques for placing centromeres on genetic maps have been developed, and a number of these methods have been used to map centromeres in maize. Maize centromeres have been mapped to regions defined by flanking molecular markers through use of B-A translocation lines (Weber and Helentjaris 1989; Lin et al. 2001) and isochromosomes produced by centric fission (Schneerman et al. 1998). Positions have been calculated from data derived from recombination between genetic markers and cytological markers (Gardiner et al. 1993; Coe and Schaeffer 2005). Half-tetrad analysis has been used to map alfalfa centromeres (Tavoletti et al. 1996) and could be used to map those of maize. Three recently developed systems could also be used to map centromeres: fluorescence in situ hybridization (FISH), recombination nodule $(\mathrm{RN})$ mapping, and radiation hybrid $(\mathrm{RH})$ lines developed from oat-maize chromosome addition lines (RieraLizarazu et al. 2000). Improvements in FISH in maize now 
permit mapping of centromeres relative to single-copy probes (Koumbaris and Bass 2003). RN mapping shares properties of genetic mapping and cytological mapping, and positions of the maize centromeres have been predicted on the $\mathrm{RN}$ map (Anderson et al. 2004). We used RH lines to map centromeres on chromosomes 1, 2, 3, 4, 6, and 9. This multitude of mapping techniques available for maize, coupled with the high-resolution genetic map (Polacco et al. 2004) and the bacterial artificial chromosome (BAC) contig map (http://www.genome.arizona.edu/fpc/maize/) make a comparison of methods possible. We therefore considered 7 maize centromeres mapped by multiple techniques in several studies. The results suggest that structural differences between chromosomes can affect our ability to map centromeres.

\section{Materials and Methods}

\section{Mapping Techniques}

Centromeres on chromosomes 1, 2, 3, 4, 6, and 9 were mapped in RH lines developed from oat-maize addition (OMA) lines. These lines were generated as described by Riera-Lizarazu et al. (2000); information about specific lines is available at http://corn.ccgb.umn.edu/. Simple sequence repeat (SSR) markers and other polymerase chain reactionbased markers were used to characterize these lines. Most markers used are described in MaizeGDB (http://www. maizegdb.org/). Primer sequences for the remaining markers (Supplementary data 1) were based on sequences placed on the BAC physical map (http://www.genome.arizona.edu/ fpc/maize/, FPC release of 19 July 2005).

Telosome and isochromosome lines were recovered by several methods. Two lines were recovered from oat-maize chromosome addition materials. One line contained a putative telosomic chromosome for the short arm of chromosome 10 (Kynast et al. 2003) and the other a putative isochromosome for the long arm of chromosome 9 (RieraLizarazu et al. 2000). Telosomes for both arms of chromosome 3 were recovered from a double-ditelosomic line (L'Heureux et al. 1997). Telotrisomics for 6L were confirmed and analyzed from these "presumptive telocentrics," and markers were placed on these materials as previously described (Schneerman et al. 1998).

Plants homozygous for the elongate-1 (el1) mutation were used to produce unreduced gametes $(2 n)$ for half-tetrad analysis. Plants homozygous for ell were crossed with the Ky21 line and then selfed. The $\mathrm{F}_{2}$ plants were pollinated by a tetraploid tester. Restriction fragment length polymorphic markers were mapped on this material as described previously (Kamps et al. 1996).

FISH was performed on pachytene chromosome spreads prepared from microsporogenic florets in meiosis taken from an OMA line plant carrying maize chromosome 9 (Koumbaris and Bass 2003). BAC clones, from a Sorghum propinquum BAC library, hybridizing to maize marker sequences were selected for use as FISH probes. These clones carried the sorghum homolog to the maize marker plus adjacent genes, which were expected to colocalize in maize and sorghum.

\section{Additional Data Sources and Integration of Mapping Data}

Additional centromere mapping data used in the comparative analysis came from studies by Weber and Helentjaris (1989), Lin et al. (1997, 2001), Schneerman et al. (1998), and Anderson et al. (2004) and from the UMC98 map (Gardiner et al. 1993) and the Genetics 2005 map (Coe and Schaeffer 2005). Supporting data for centromere positions in the UMC98 and Genetics 2005 maps are available at http:// www.maizegdb.org. The IBM2 (intermated B73 × Mo17) neighbors 2004 map was used as the reference map for our study (Polacco et al. 2004). This map used the highresolution IBM2 genetic map for a framework; markers not mapped on the IBM2 genetic map were integrated into this framework to produce the IBM2 neighbors 2004 map. When centromere positions were assigned, greater weight was given to markers that had been mapped on the 302member IBM2 mapping population. Some conflicts in marker order between the RH mapping data and genetic maps were resolved with the help of the BAC physical map (http://www.genome.arizona.edu/fpc/maize/).

To facilitate comparisons between chromosomes, we report map positions as the fractional distance from the tip of the short arm. For example, the chromosome 9 centromere was placed between umc2337 on the short arm and csu623 on the long arm. Markers umc2337, at map position 220.10, and csu623, at position 230.60, were used to define the centromere location on the genetic map. These 2 markers were mapped using the IBM2 population and represent the best available genetic mapping data. The total length of the IBM2 neighbors 2004 chromosome 9 map was $846.64 \mathrm{cM}$, from -41.50 to 805.14. Therefore, the centromere resides between 0.309 and 0.321 of the genetic distance from the tip of the short arm along chromosome 9.

\section{Results}

\section{Mapping Centromere Positions from Induced Chromosome Breaks}

Results from 2 pairs of $\mathrm{RH}$ lines placed the chromosome 9 centromere between bnlg127 and csu623. Line RH9.01.3001.2-01 carried markers on the long arm, and line RH9.01.1-027.2-03 carried markers from the short arm (Figure 1; Supplementary data 2). Because the maize centromere repeat sequence, CentC (Ananiev et al. 1998), was not detected in either line by Southern blot analysis (data not shown), the centromere probably lies between bnlg127 and csu623. These 2 lines probably represent translocations between the maize chromosome and the oat chromosome. This result placed the centromere between BAC contigs 375 and 376 on the BAC physical map. Data from a second pair of lines, RH9.01.3-001.2-02 and RH9.01.1-069.4-12, supported the placement of the centromere between bnlg127 and csu623. Both lines had chromosome breaks in the 
Chromosome 6
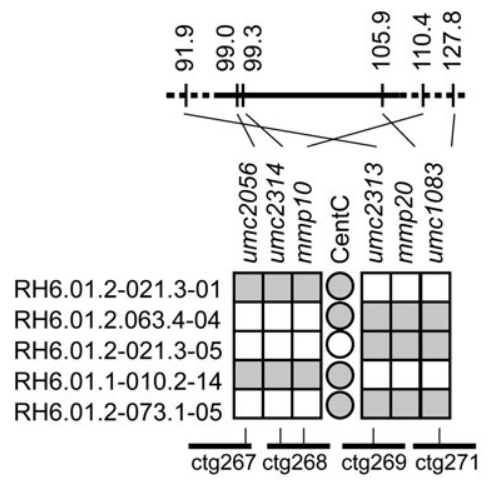

Chromosome 9

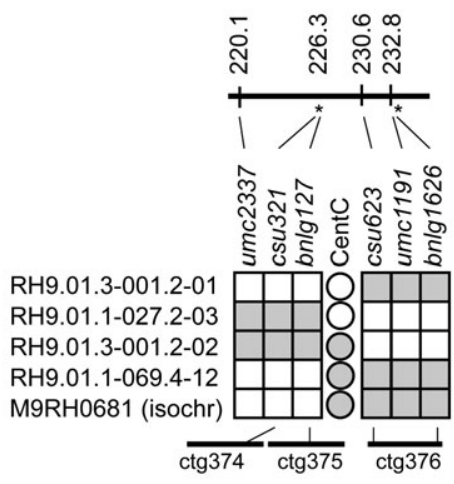

Chromosome 10

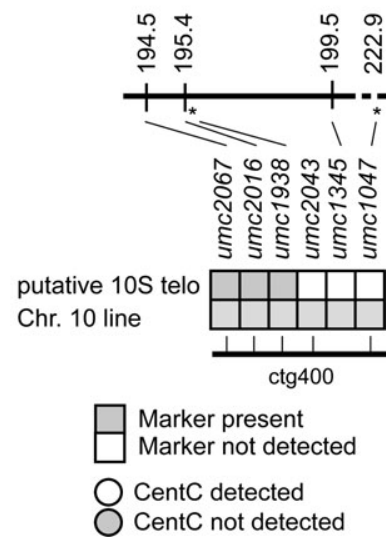

Figure I. Mapping centromeres in OMA materials. Marker data from RH lines, a chromosome 9 isochromosome line, and a chromosome 10 pseudocentric fission line were aligned with the IBM2 neighbors 2004 map (above) and the BAC contig map (below). Markers indicated with asterisks were not mapped on the full IBM2 population and may not be as accurately placed as those mapped on the IBM2 population. However, the discrepancy between the map positions of mmp10 and umc2313 on chromosome 6 illustrates the difficulties in reconciling mapping data. The presence of a maize centromere in an RH line was determined by Southern blot analysis using a Cent $C$ centromere repeat sequence probe.

interval between bnlg127 and csu623 and retained CentC sequences and hence the maize centromere. Basing centromere positions on several independent lines increased the confidence of mapping by minimizing the possibility that small internal deletions or other rearrangements in an $\mathrm{RH}$ line confounded marker analysis. Markers umc2337 and csu623 were chosen to anchor the centromere on the genetic map. These 2 markers were mapped on the full IBM2 population, unlike csu321 and bnlg127, and represent the best genetic mapping data. Map positions of centromeres on chromosomes 1, 2, 3, 4, and 6 were determined similarly (Figure 1, Table 1; Supplementary data 2).

Discrepancies in marker order among the genetic map, BAC physical map, and RH lines were found on some chromosomes. Small differences can reasonably be attributed to statistical error. Others, like the larger inversion of mmp10 and umc2313 on chromosome 6 were less likely to be the result of statistical error and may represent small chromosome rearrangements or alternative positions for individual markers.

\section{Mapping Centromeres by Means of Spontaneous Chromosome Breaks}

A chromosome 9 putative isochromosome and a chromosome 10 putative telosome were recovered from OMA lines. The work of Jin et al. (2004) has shown that the functional centromere on maize chromosomes present in OMA lines colocalize with Cent $C$ centromere repeat sequences. These results argue that centric fission of maize chromosomes in OMA lines probably occurs at the normal maize centromere. Cytological observations and marker data of the chromosome 9 putative isochromosome line M9RH0681 had suggested that this line carried 2 copies of the long arm fused at the centromere (Riera-Lizarazu et al. 2000). Additional marker work placed the chromosome 9 centromere between bnlg127 and csu623, the same interval that was identified from RH lines (Figure 1).

The chromosome break in the putative chromosome 10 telosome (Kynast et al. 2003) was mapped to BAC contig 400 between markers umc1938 and umc2043 (Figure 1). If contig 400 carried the centromere, then this contig should carry a large block of Cent $C$ centromere repeat sequences, centromeric repetitive elements, and few genes. Instead, many gene sequences have been detected in this contig but no Cent $C$ sequence (http://www.genome.arizona.edu/fpc/ maize/). The absence of the block of Cent $C$ sequence means that contig 400 is unlikely to contain a maize centromere. Thus, the putative chromosome 10 telosome seems to have been created by a spontaneous chromosome break on the long arm near the centromere. Such events, known as pseudocentric fission events, have been described in other organisms (Perry et al. 2005). This example illustrates the dangers of basing a centromere position on a single line.

Centric fission products from maize plants for chromosomes 3 (L'Heureux et al. 1997) and 6 (Doyle GG, unpublished data) were used to map centromeres. Telocentrics for both arms of chromosome 3 were used to locate the chromosome 3 centromere. The structure of these telosomes was supported by examination of pachytene chromosomes (data not shown). The most proximal marker on the shortarm telosome detected by Southern blot analysis was umc10a (not placed on a BAC contig), and umc102 (BAC contig 124) was the most proximal marker detected on the long arm (Figure 2). These results placed the centromere between positions 259.40 and 296.10 on the IBM2 neighbors 2004 map. The centromere position on chromosome 6 was determined on 2 long-arm telocentric chromosomes, telo-6La 
Table I. Comparison of centromere locations in different studies ${ }^{a}$

\begin{tabular}{|c|c|c|c|c|c|c|c|}
\hline & Chr I & Chr 2 & Chr 3 & Chr 4 & Chr 6 & Chr 9 & Chr 10 \\
\hline $\mathrm{B}-\mathrm{A}^{b}$ & $\begin{array}{l}0.330-0.452 \\
(\text { sod4-npi304 })\end{array}$ & $\begin{array}{l}0.417-0.478 \\
(\text { npi242a-npi123 }\end{array}$ & $\begin{array}{l}0.211-0.267 \\
\text { (npi446-npi247) }\end{array}$ & $\begin{array}{l}0.471-0.591 \\
\text { (npi259-umc19) }\end{array}$ & $\begin{array}{l}0.119-0.154 \\
\text { (npi235-npi377) }\end{array}$ & $\begin{array}{l}0.275-0.320 \\
\left(w \times 1-b n l 5.10^{\circ}\right)\end{array}$ & 0.336-0.364(npi105a-glu1) \\
\hline $\mathrm{B}-\mathrm{A}^{f}$ & $\begin{array}{l}0.463-0.496 \\
(\text { umc67a-umc177a) }\end{array}$ & ND & ND & ND & $\begin{array}{l}0.131-0.130 \\
(\text { bnl6.29a-bnl7.28 }\end{array}$ & $\begin{array}{l}0.320-0.343 \\
(\text { bnl5.10 }- \text { umc } 20)\end{array}$ & ND \\
\hline RH map ${ }^{b}$ & $\begin{array}{l}0.414-0.416 \\
\text { (umc1461-mmp101) }\end{array}$ & $\begin{array}{l}0.455-0.455 \\
(\text { umc1635-umc1581 })\end{array}$ & $\begin{array}{l}0.303-0.477 \\
(\text { AY110297-umc1730) }\end{array}$ & $\begin{array}{l}0.465-0.473 \\
(\text { umc1953-umc1142) }\end{array}$ & $\begin{array}{l}0.176-0.187 \\
(\text { umc2314-umc2313) }\end{array}$ & $\begin{array}{l}0.309-0.321 \\
(\text { umc2337-csu623a) }\end{array}$ & ND \\
\hline Centric fission ${ }^{j}$ & ND & ND & $\begin{array}{l}0.327-0.370 \\
(\text { umc10a-umc102) }\end{array}$ & $\begin{array}{l}0.463-0.468 \\
(b n l 5.45-b n l 7.20)\end{array}$ & $\begin{array}{l}0.140-0.175 \\
(\text { umc59a-csu146a) }\end{array}$ & $\begin{array}{l}0.309-0.321 \\
(\text { umc2337-csu623a) }\end{array}$ & $\begin{array}{l}0.394-0.401 \\
(\text { umc2016-umc1345) }\end{array}$ \\
\hline $\mathrm{UMC}^{k} 8^{k}$ & 0.521 & 0.456 & 0.378 & 0.466 & 0.127 & 0.304 & 0.364 \\
\hline Genetics $2005^{l}$ & 0.433 (eno2) & 0.45 (umc131, umng2) & $0.294(\operatorname{csu} 1070)$ & 0.465 (csu716, agrp83a) & $0.174(\operatorname{csu} 1120, \text { uck. } 1)^{g}$ & $\begin{array}{l}0.299 \\
(\text { umc } 273 a, \text { csu680d) }\end{array}$ & $\begin{array}{l}0.364 \\
(p h p 06005, \text { rgpc1122d) }\end{array}$ \\
\hline $\mathrm{RN} \operatorname{map}^{m}$ & $\begin{array}{l}0.496 \\
\text { (asg } 58 \text {-csu805) }\end{array}$ & 0.456 (umng1) & $\begin{array}{l}0.400 \\
(\text { umc102a-umc26a) }\end{array}$ & $0.466(b n l 5.45-u m c 156 a)$ & $\begin{array}{l}0.129 \\
(u m c 85 a-b n \lg 426)\end{array}$ & $0.304(w \times 1-u m c 81)$ & $\begin{array}{l}0.368 \\
(p h p 06005-p h p 20646)\end{array}$ \\
\hline $\mathrm{FISH}^{b}$ & ND & ND & $\mathrm{ND}$ & ND & ND & $\begin{array}{l}0.275-0.316 \\
(w \times 1-c d 017)\end{array}$ & ND \\
\hline Half-tetrad $^{b}$ & ND & 0.459 & ND & ND & ND & ND & ND \\
\hline$r-X 1^{n}$ & ND & $\begin{array}{l}0.452-0.497 \\
(\text { umc131-umc55a) }\end{array}$ & ND & ND & $0.00-0.130$ to $b n l 7.28^{\circ}$ & ND & $0.00-0.598$ to $\operatorname{csu} 6 b^{\circ}$ \\
\hline $\begin{array}{l}\text { IBM2 neighbors } \\
2004 \text { map }\end{array}$ & $\begin{array}{l}1193.70 \\
(-55.80 \text { to } 1137.90)\end{array}$ & $\begin{array}{l}781.67 \\
(-11.07 \text { to } 770.60)\end{array}$ & $\begin{array}{l}879.33 \\
(-21.30 \text { to } 858.03)\end{array}$ & $\begin{array}{l}954.03 \\
(-149.20 \text { to } 804.83)\end{array}$ & $\begin{array}{l}581.94 \\
(-2.90 \text { to } 579.04)\end{array}$ & $\begin{array}{l}846.64 \\
(-41.50 \text { to } 805.14)\end{array}$ & $\begin{array}{l}557.20 \\
(-24.00 \text { to } 533.20)\end{array}$ \\
\hline
\end{tabular}

ND, not determined.

${ }^{a}$ Centromere positions expressed as fractional distance along the genetic map from the tip of the short arm.

${ }^{b}$ Data from Weber and Helentjaris (1989).

${ }^{c}$ Markers indicated were the ones chosen to place the centromere.

${ }^{d} n$ pi123c was used to place the centromere, rather than npi123a (also known as $n p i 47 a$ ), which is more distal on the long arm.

${ }^{e}$ bnl5.10 was placed on the long arm by Weber and Helentjaris (1989) and on the short arm by Lin et al. (2001).

${ }^{f}$ Data from Lin et al. (2001).

${ }^{g}$ The order of several markers around the centromere is inverted.

${ }^{b}$ Data presented here.

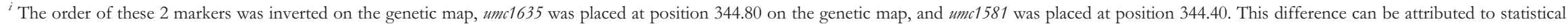
errors, and the order of the markers assumed in our study was consistent with the BAC physical map and the RH map.

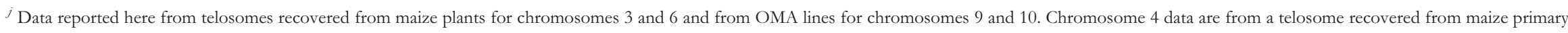
trisomics in Schneerman et al. (1998).

${ }^{k}$ Gardiner et al. (1993).

' Coe and Schaeffer (2005); centromere positions were either based on a marker mapped on top of the centromere or interpolated from 2 flanking markers.

"m Anderson et al. (2004).

${ }^{n}$ Lin et al. (1997).

${ }^{\circ}$ Data from chromosome breaks on long arm only. 

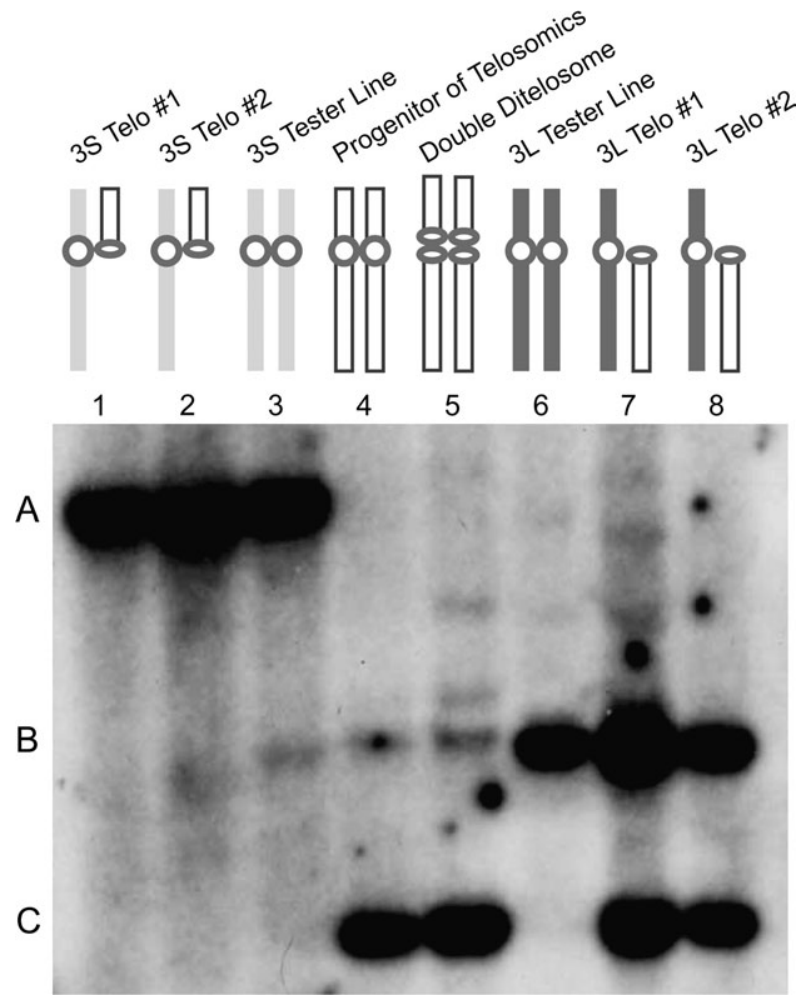

Figure 2. Placement of umc102a on 3L in Telo3 lines. "A" indicates the band contributed by the $3 \mathrm{~S}$ tester line, " $\mathrm{B}$ " indicates the contribution from the $3 \mathrm{~L}$ tester, and " $\mathrm{C}$ " indicates the band contributed by the telosome progenitor. Lane 4 shows the fragment detected by the umc102a probe from the progenitor of the telosomic materials, and lane 6 presents the fragment from the tester line used to recover $3 \mathrm{~L}$ telosomes. The presence of both fragments in the 2 long-arm telosomes, lanes 7 and 8 , demonstrates that umc102a sequences were present on the long arm of chromosome 3. Sequences of umc102a were not detected on the short arm, lanes 1 and 2 .

and telo-6Lc. Southern blot analysis detected the marker csu146a on telo-6La and telo-6Lc but not umc59a (data not shown). These results placed the centromere proximal to csu146a on the long arm, and because this was a centric fission, umc59a must reside on the short arm. On the BAC physical map, the order of csu146a and umc59a was inverted and was inconsistent with results from telo-6La and telo6Lc. Probes used to detect umc59a mapped to several locations on genetic maps (http://www.maizegdb.org/) and to several contigs on the BAC physical map. The sequence reported as umc59a on the BAC physical map may correspond to a related sequence.

\section{Half-Tetrad Analysis}

Barrell and Grossniklaus (2005) have argued, from their cytological work, that unreduced gametes from el1/el1 plants are exclusively the product of meiotic restitution by omission of the second meiotic division. Such gametes are
Production of $2 \mathrm{~N}$ gametes in elongate 1

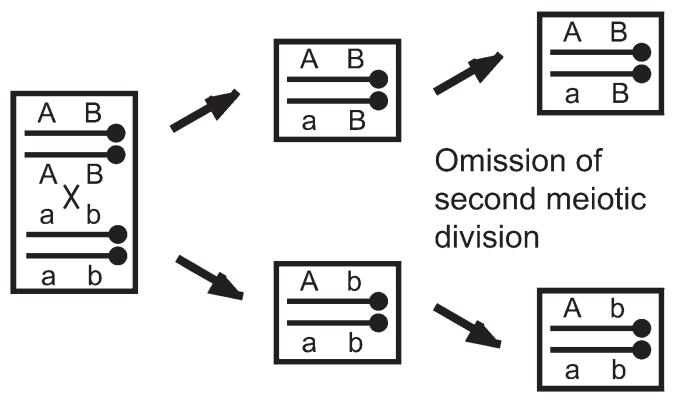

Figure 3. Segregation of alleles in unreduced $e l 1$ gametes. The $2 n$ gametes are homozygous for markers close to the centromere unless a crossover occurs between the marker and the centromere.

homozygous for markers tightly linked to the centromere (Figure 3). Markers farther from the centromere may be heterozygous in unreduced gametes, and the frequency of heterozygosity will depend on the distance to the centromere. This trend was observed for markers b1, bnl10.42, bnl12.09, and npi452 (Supplementary data 3).

Tetraploid progeny derived from diploid el1/ell plants crossed with tetraploid testers were genotyped for markers on chromosome 2. For the bnl12.09 locus, 11 progeny were heterozygous for the el1 and the Ky21 alleles. Crossovers therefore occurred between bnl12.09 and the centromere in 11 of the 60 plants tested, and the distance from bnl12.09 to the centromere was approximately $18 \mathrm{cM}$. A map position of 77.00 on the UMC98 map for bnl12.09 was interpolated from the flanking markers prp2 and csu56c, placing the centromere at 95.00. Transferring this position to the IBM2 neighbors 2004 map placed the centromere at position 348.00 .

\section{FISH Analysis}

Seven FISH probes spanning the centromere region were used to place the chromosome 9 centromere (Supplementary data 4). Two of the sorghum BACs used as FISH probes were detected on the short arm (Figure 4). These $\mathrm{BAC}$ clones contained sequences homologous to $w \times 1$ and tda66d. Wx1 placed the centromere proximal to position 191.70. The most proximal marker on the long arm was cdo17, at position 212.80. This map position may not be accurate because $c d 017$ and other markers on the long arm were not mapped with the IBM2 population. Csu321, placed on the long arm by FISH, had been placed on the short arm by RH mapping.

\section{Discussion}

The combination of complete genome sequences from key species and the conservation of gene order in related species are powerful tools for predicting the location of genes. 


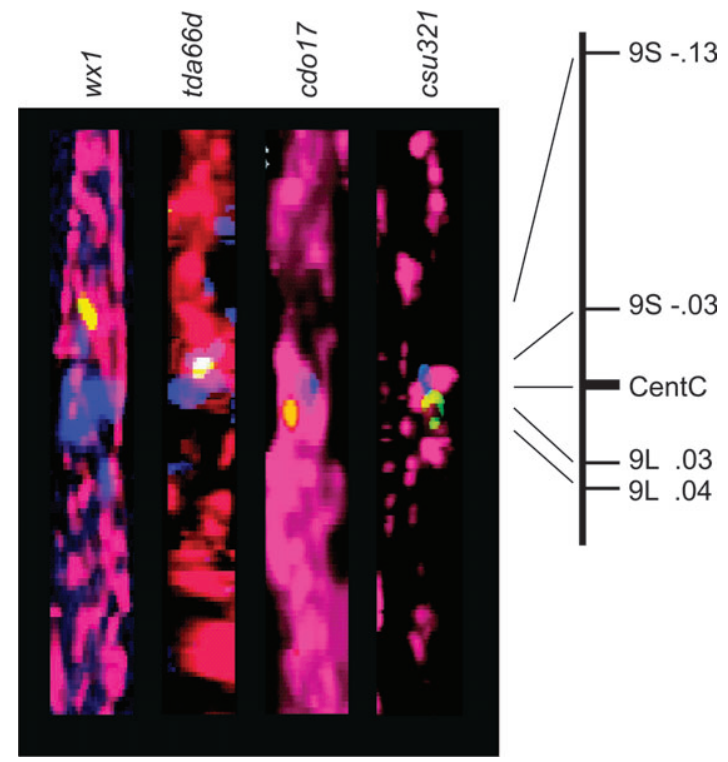

Figure 4. Mapping centromere 9 by FISH. Straightened projections of maize chromosome 9 showing the sorghum BAC FISH probe signals (green/yellow) for $w \times 1, t d a 66 d$, cdo17, and csu321 and the centromere Cent $C$ repeat (blue/purple). The split signal for $t d a 66 d$ was occasionally seen with this and other probes. The map positions of markers on the cytological map were based on 35 or more chromosomes per marker. The positions of the markers on the cytological map are shown on the right; $w \times 1$ is on chromosome 9 at position 0.13 on the short arm.

However, positions of some maize centromeres do not appear to correspond to the locations of ancestral centromeres from rice. The centromere on maize chromosome 4 may have originated after rice and maize diverged as the region containing this centromere corresponds to central regions on the long arms of rice chromosomes 2 and 4 (http://www.agcol.arizona.edu/symap/). Unfortunately, the alignments between maize and rice near the centromeres were usually poor making comparisons difficult. In mammals, centromere repositioning has been observed in homologous chromosomes from related species even though gene order is conserved. For instance, gene order is apparently conserved along the $\mathrm{X}$ chromosomes from humans, ringtailed lemurs, and black lemurs, but the centromere positions range from the middles to the tips of the chromosomes (Ventura et al. 2001). Change in centromere positions over time, coupled with the tendency for centromeres to fall within gaps in genome sequences, may preclude the accurate prediction of centromere locations even when high-quality genome sequences are available.

\section{Comparisons of Mapping Techniques}

Centromere locations determined here from centric fission lines and induced chromosome breaks in RH lines were consistent with reported centromere positions from similar studies for chromosomes 2, 4, and 9 (Table 1). For example, on chromosome 2, $\mathrm{RH}$ mapping placed the centromere between umc1635 and umc1581. Both markers were located at a relative position of 0.455 , where 0.455 represents the map position as the fraction of the distance from the tip of the short arm along the IBM2 neighbors 2004 genetic map. This centromere was supported by mapping studies using BA translocation lines (Weber and Helentjaris 1989) and with $r$-X1 deficiency lines (Lin et al. 1997) (Table 1). The less precise positioning of centromeres in studies using B-A translocation terminal deficiency lines is probably because chromosome breaks were at a considerable distance from the centromere.

Results of studies of centromere position based on centric fission events and induced chromosome breaks were not consistent for chromosomes 1,3 , and 6 (Table 1). RH mapping placed the chromosome 1 centromere between umc1461 and mmp101. This location was consistent with B-A translocation data from Lin et al. (2001) but not with those from Weber and Helentjaris (1989). Results from centric fission lines and RH lines were consistent for chromosome 3 , but they conflicted with those of Weber and Helentjaris (1989). On chromosome 6, results from RH mapping were inconsistent with both B-A translocation studies (Weber and Helentjaris 1989; Lin et al. 2001) and a study using terminal deficiencies (Lin et al. 1997). These differences could indicate real differences in centromere positions, or they may reflect the use of poorly mapped markers or undetected rearrangements in mapping materials. If technical problems lie at the root of differences in centromere positions, perhaps, additional studies using different techniques and materials would resolve such discrepancies in centromere positions.

Centromeres can be genetically mapped directly by halftetrad analysis or mapped using translocation lines. The strength of half-tetrad analysis is that the functional centromere is mapped. Other approaches require assumptions such as the colocalization of centromeres with Cent $C$ repeat sequences. Unfortunately, attempts to map centromeres on chromosomes 3 and 9 were hindered by the difficulty of identifying SSR markers polymorphic in both parent lines and the tetraploid testers used to recover unreduced gametes. Half-tetrad analysis accurately mapped the chromosome 2 centromere, but data were insufficient to permit a comparative evaluation of half-tetrad mapping. Genetic mapping of centromeres relative to translocation breakpoints placed centromeres on the UMC98 map (Gardiner et al. 1993) and the Genetics 2005 map (Coe and Schaeffer 2005). On chromosomes 2, 4, 9, and 10, centromere positions were consistent on the UMC98 and Genetics 2005 maps and with centromere positions determined by other methods. Centromere positions on the UMC98 and Genetics 2005 maps for chromosomes 1, 3, and 6 differed. Thus, conflicting centromere positions were likewise found on the same chromosomes by genetic mapping and by mapping based on spontaneous and induced chromosome breaks (Table 1).

Because much of the genetic mapping data used to place centromeres were derived from B-A translocations and because some of the same mapping data were used in both 

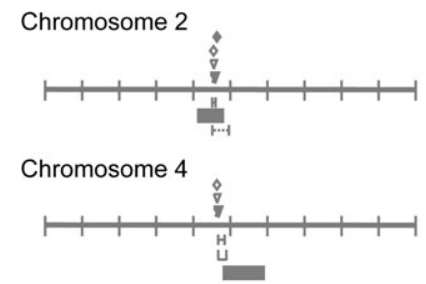

Chromosome 9

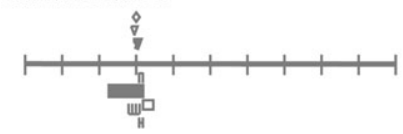

Chromosome 10

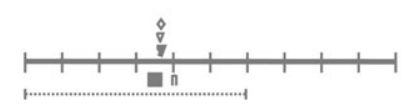

Chromosome 1

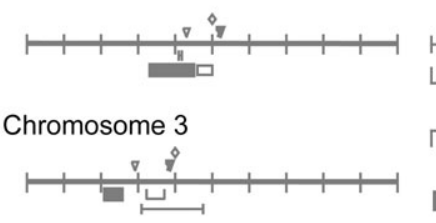

Chromosome 6

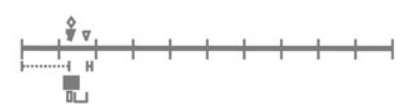

$\longmapsto$ Radiation hybrid mapping (here)

$\sqcup$ Centric fission-maize (Schneerman et al., 1998, and here)

$\square$ Centric fission- OMA (here)

- Half-tetrad analysis (here)

B-A translocations (Weber and Helentjaris, 1989)

ш FISH (here)

$\square$ B-A translocations (Lin et al., 2001)

$\diamond$ Recombination nodule map

(Anderson et al., 2004)

$\nabla$ UMC98 Map (Davis et al., 1999)

$\nabla$ Genetics 2005 Map (Coe and Schaeffer, 2005)

r--- r-X1 induced deletions (Lin et al., 1997)

Figure 5. Composite centromere map data. The difference between chromosomes 2, 4, 9, and 10 and chromosomes 1,3 , and 6 were apparent when data from centromere mapping studies were placed on common maps. Map positions are presented as the fractional distances along the IBM2 neighbors 2004 maps.

the UMC98 map and the Genetics 2005 map, an independent source of genetic mapping data was considered. RN mapping is based on the number of crossover events observed along chromosomes by electron microscopy. Centromere positions were calculated from the genetic distance from telomeres to centromeres and then aligned along the UMC98 map (Anderson et al. 2004). Centromere locations were then placed on the reference map and flanking markers used to compare $\mathrm{RN}$ mapping with other techniques. As with other techniques, centromere positions determined by $\mathrm{RN}$ mapping were consistent with other reported centromere positions on chromosomes 2, 4, 9, and 10 (Table 1). The RN map was based on a large sample of approximately 200 meiotic events for each chromosome, so large errors in map positions were unlikely (Anderson et al. 2004). We noted no obvious problems with the RN mapping technique and therefore no reason why the centromere positions on chromosomes 1, 3, and 6 should be doubted.

Similarly, support for centromere positions based on $\mathrm{RH}$ mapping was strong. Between 2 and 5 independent chromosome break events were used to locate centromeres on the RH maps. Multiple independent rearrangements would have been necessary to place centromeres in the wrong region, and poorly mapped markers were also unlikely to have affected mapping results. We aligned markers used in this work on the IBM2 high-resolution genetic map, the BAC physical map, and the RH map to reduce this possibility (Figure 1; Supplementary data 2). Nonetheless, centromere positions based on RH maps and $\mathrm{RN}$ maps did not agree for chromosomes 1 and 6 .

The confidence with which a maize centromere could be mapped depended on the chromosome and not on the technique used. Chromosomes 1 and 2 illustrate this conclusion (Figure 5, Table 1). The chromosome 2 centromere position was consistent in 7 studies using 6 techniques. In contrast, for chromosome 1, 2 B-A translocation studies disagreed (Weber and Helentjaris 1989; Lin et al. 2001), as did 2 retrospective genetic analyses (Gardiner et al. 1993; Coe and
Schaeffer 2005). The centromere position on the RH map was consistent with one retrospective genetic analysis and one B-A translocation study, whereas the centromere position predicted by the RN map was consistent with the other retrospective genetic analysis and B-A translocation study. Results from any single centromere mapping study may place the centromere at a location inconsistent with other studies.

These results do not imply that all techniques are equally accurate. First, the resolution for centric fission lines and $\mathrm{RH}$ lines was usually better than that for other techniques, although the effort required to recover centric fission lines and RH lines is considerable. Second, studies using the same technique are not necessarily equal. The B-A translocation study by Weber and Helentjaris (1989) relied on more markers and lines and can be considered more reliable than that of Lin et al. (2001), who used fewer.

\section{Do Some Maize Chromosomes Have Alternative Centromere Positions?}

The appearance of a neocentromere accompanied by the inactivation of the normal centromere on human chromosome 4 raises the possibility of alternative centromere positions (Amor et al. 2004). Transmission of this neocentromere has been traced over 3 generations, so heterozygosity for a normal chromosome and a chromosome with a neocentromere can be tolerated. Our study provides evidence for alternative centromere positions in some maize chromosomes. RH mapping, B-A translocations (Weber and Helentjaris 1989), and the Genetics 2005 map (Coe and Schaeffer 2005) provided good support for a chromosome 1 centromere position near position 0.420, whereas RN mapping (Anderson et al. 2004), B-A translocations (Lin et al. 2001), and the UMC98 map (Gardiner et al. 1993) provided good support for a position near 0.500 . Alternative centromere locations of 0.130 and 0.180 on chromosome 6 were also supported. The possibility that centromeres can reside in more than one 
place on maize chromosomes was raised by studies describing 2 blocks of the CentC centromere repeat sequence on chromosome 10 (Wang et al. 2006) and by differences in the locations of centromeres on chromosomes, as determined by arm ratios (Neuffer et al. 1997). The observation of a pericentric inversion by McClintock (1933) provides another mechanism for moving centromeres, one that does not rely on the formation of neocentromeres.

Variation in centromere position may also exist for chromosome 9. RH mapping placed csu321 on the short arm, but FISH placed it on the long arm. RH lines were derived from the OMA9.01 line, whereas the OMA9.02 line was used for FISH studies. The 2 OMA lines shared the Seneca 60 maize parent (Kynast et al. 2001), but Seneca 60 is a hybrid, and SSR marker analysis revealed that the centromere regions in the OMA9.01 and OMA9.02 lines came from the different lines used to produce the hybrid (data not shown). The causes of discrepancies in centromere map positions have not been resolved, but differences in the structure of centromere regions between lines can lead to real differences in the location of maize centromeres or apparent differences in centromere locations due to differences in marker positions. New approaches to centromere mapping may allow the efficient mapping of centromeres in diverse backgrounds (Luce et al. 2006). A closer examination of diverse maize lines may reveal a rich diversity in centromere regions.

\section{Supplementary Material}

Supplementary data 1-4 can be found at http://www.jhered. oxfordjournals.org/.

\section{Funding}

National Science Foundation (0110134 to R.L.P. and H.W.R. and DBI-0321639 to H.W.B.); United States Department of Agriculture-Agricultural Research Service (58-3622-1-133 to Ed Coe).

\section{Acknowledgments}

We thank Jeff Bennetzen for providing the Sorgbum bicolor BAC clone, bWx1-131-L1, Herika Zaia for help in developing materials, Matt Walch for reading sections of the manuscript, and Anne Thistle for editorial assistance. We also thank Ed Coe for his insights and for his many years devoted to mapping the maize genome.

\section{References}

Amor DJ, Bentley, Ryan J, Perry J, Wong L, Slater H, Choo KHA. 2004. Human centromere repositioning "in progress." Proc Natl Acad Sci USA. 101:6542-6547.

Ananiev EV, Phillips RL, Rines HW. 1998. Chromosome-specific molecular organization of maize (Zea mays L.) centromeric regions. Proc Natl Acad Sci USA. 95:13073-13078.
Anderson LK, Salameh N, Bass HW, Harper LC, Cande WZ, Weber G, Stack SM. 2004. Integrating genetic linkage maps with pachytene chromosome structure in maize. Genetics. 166:1923-1933.

Barrell PJ, Grossniklaus U. 2005. Confocal microscopy of whole ovules for analysis of reproductive development: the elongate 1 mutant affects meiosis II. Plant J. 43:309-320.

Coe EH, Schaeffer ML. 2005. Genetic, physical, maps, and database resources for maize. Maydica. 50:285-303.

Gardiner J, Coe EH, Melia-Hancock S, Hoisington DA, Chao S. 1993. Development of a core RFLP map in maize using an immortalized F2 population. Genetics. 134:917-930.

Jin W, Melo JR, Nagali K, Talbert PB, Henikoff S, Dawe RK, Jiang J. 2004. Maize centromeres: organization and functional adaptation in the genetic background of oat. Plant Cell. 16:571-581.

Kamps TL, McCarty D, Chase CD. 1996. Gametophyte genetics in Zea mays L: dominance of a restoration-of-fertility allele $(R f 3)$ in diploid pollen. Genetics. 142:1001-1007.

Koumbaris GL, Bass HW. 2003. A new single-locus cytogenetic mapping system for maize (Zea mays L.): overcoming FISH detection limits with marker-selected sorghum (S. propinquum L.) BAC clones. Plant J. 35:647-659

Kynast RG, Okagaki R, Granath S, Rines H, Phillips RL. 2003. A novel maize ditelosome 10 addition to oat cv. Sun II for use in radiation hybrid mapping. Maize Genet Coop News Lett. 77:62-63.

Kynast RG, Riera-Lizarazu O, Vales MI, Okagaki RJ, Maquieira SB, Chen G, Ananiev EV, Odland WE, Russell CD, Stec AO, et al. 2001. A complete set of maize individual chromosome additions to the oat genome. Plant Physiol. 125:1216-1227.

L'Heureux TA, Muzumdar DA, Schneerman MC, Weber DF. 1997. Haploids and monosomics are produced by maize plants containing telocentrics for both arms of chromosome 3 and B chromosomes. Maize Genet Coop News Lett. 71:66-67.

Lin B-Y, Chang S-J, Lin H-M. 2001. Centromere position in maize. Bot Bull Acad Sin. 42:273-279.

Lin B-Y, Peng S-F, Chen Y-J, Chen H-S, Kao C-F. 1997. Physical mapping of RFLP markers on four chromosome arms in maize using terminal deficiencies. Mol Gen Genet. 256:509-516.

Luce AC, Sharma A, Mollere OSB, Wolfgruber TK, Nagaki K, Jiang J, Presting GG, Dawe RK. 2006. Precise centromere mapping using a combination of repeat junction markers and chromatin immunoprecipitation-polymerase chain reaction. Genetics. 174:1057-1061.

McClintock B. 1933. The association of non-homologous parts of chromosomes in the mid-prophase of meiosis in Zea mays. Z Zellforsch Mikrosk Anat. 19:191-237.

Neuffer MG, Coe EH, Wessler SR. 1997. Mutants of maize. New York: Cold Spring Harbor Laboratory Press.

Perry J, Nouri S, La P, Daniel A, Wu Z, Purvis-Smith S, Northrop E, Choo KHA, Slater HR. 2005. Molecular distinction between true centric fission and pericentric duplication-fission. Hum Genet. 116:300-310.

Polacco ML, Sanchez-Villeda H, Coe E. 2004. IBM neighbors-mutual enhancement of genetic and physical maps. Maize Genet Conf Abstr. 46:P110.

Riera-Lizarazu O, Vales MI, Ananiev EV, Rines HW, Phillips RL. 2000. Production and characterization of maize chromosome 9 radiation hybrids derived from an oat-maize addition line. Genetics. 156:327-339.

Schneerman MC, Lee WS, Doyle G, Weber DF. 1998. RFLP mapping of the centromere of chromosome 4 in maize using isochromosomes for $4 \mathrm{~S}$. Theor Appl Genet. 96:361-366.

Tavoletti S, Bingham ET, Yandell BS, Veronesi F, Osborn TC. 1996. Half tetrad analysis in alfalfa using multiple restriction fragment 
length polymorphism markers. Proc Natl Acad Sci USA. 93:1091810922.

Ventura M, Archidiacono N, Rocchi M. 2001. Centromere emergence in evolution. Genome Res. 11:595-599.

Wang C, Harper LC, Cande WZ. 2006. High-resolution single-copy gene fluorescence in situ hybridization and its use in the construction of a cytogenetic map of maize chromosome 9. Plant Cell. 18:529-544.
Weber D, Helentjaris T. 1989. Mapping RFLP loci in maize using B-A translocations. Genetics. 121:583-590.

Received April 24, 2007

Accepted September 25, 2007

Corresponding Editor: Ernest Bailey 